\title{
Prevalence of myocardial ischaemia among diabetics determined by validated Sinhala version of the WHO Rose angina questionnaire
}

\author{
Liyanage PLGC ${ }^{1}$, Sathananthan PP $^{2}$ \\ ${ }^{\prime}$ Department of Medicine, Faculty of Medicine, University of Ruhuna, Galle, Sri Lanka \\ ${ }^{2}$ Teaching Hospital Karapitiya, Galle, Sri Lanka
}

Correspondence: Dr.P.L.G.C. Liyanage (gayanicl@yahoo com)

\begin{abstract}
Objectives: The aim of this study was to validate the original version of the Rose Angina Questionnaire (RAQ) and apply it to a group of long standing diabetics in a cross-sectional manner to assess it applicability in clinical settings.

Design Setting: After a face validation and piloting the Part A of the Sinhala translated RAQ (SRAQ-Part A) was given to 47 adult patients who presented with chest pain where the possibility of stable angina was considered in the differential diagnosis. In these patients, the diagnosis of stable angina was confirmed by resting ECG. Patients who had a normal resting ECG or changes non-confirmatory of myocardial ischaemia underwent treadmill examination to detect exercise-induced myocardial ischaemia.

Part B of the translated RAQ (SRAQ-Part B) was given to 68 patients who presented with acute chest pain of less than 24 hours where acute coronary syndrome was considered a possibility. These patients underwent serial ECGs and cardiac enzymes/troponin estimations.

The validated SRAQ were administered to a group of diabetics $(n=311)$ attending medical clinics of Teaching hospital, Karapitiya in a cross-sectional manner to detect the prevalence of stable angina or history of acute coronary syndrome.

Results: Sensitivity, specificity, positive predictive value, and negative predictive values of SRAQ-Part A were $86.4 \%, 76 \%, 76 \%, 86 \%$ and of part-B were $74.1,70 \%, 93.5 \%, 31.8 \%$, respectively.

Of 311 diabetic patients the prevalence of stable angina in this group of patients would be $31 \%(95 \%$ $\mathrm{CI}=26-37 \%$ ). Part B of the questionnaire, 17 answered positively and rest negatively. Of these 17 positives, 16 were likely to have acute coronary disease.

Interpretation: Our analysis shows that the Sinhala translation of the RAQ has an acceptable specificity and sensitivity in detecting either stable angina or acute coronary syndrome. The prevalence of stable angina was $31 \%$ among the diabetics selected for this analysis.
\end{abstract}

Keywords: Validation, Questionnaire, Angina, Diabetes mellitus

\section{Introduction}

The Rose Angina Questionnaire (RAQ) is widely used as a validated tool for detecting different forms of myocardial ischaemia in population surveys since 1962 (1). The questionnaire uses the definition of angina as a "chest pain which limits exertion, situated over the sternum or left chest or left arm and is relieved within 10 minutes by rest" (1). RAQ has been used to study the natural course of ischaemic heart disease, monitor the response to interventions and to compare the prevalence of myocardial ischaemia in different populations (2-4). The questionnaire has been translated in many other languages in Western countries and also in Asia $(3,5,6)$. 
The unavailability of a gold-standard to detect myocardial ischaemia in community setting is a practical limitation when validating the RAQ. Previous studies have used many reference standards such as expert clinical diagnosis, electrocardiography (ECG), coronary angiography and exercise thallium scans as the reference standards (1, 5-7). The accuracy, availability and practicality of these diagnostic tools, however, vary widely.

Although there are no uniformly agreed cut-off values of sensitivity and specificity required for a screening tool, these values should be at an acceptable level to minimize false positives and negatives. A previous attempt to validate a modified version of the RAQ in Sri Lanka has resulted in a low specificity $(31.3 \%)$ although the sensitivity was at an acceptable level (80\%) (8). Application of this version in a community setting could be limited as it has a low positive likelihood ratio (1.2) and the posttest probability of myocardial ischaemia in a test positive person living in a setting associated with low myocardial ischaemia prevalence, would not be high enough.

Therefore we wished to validate the original version of the RAQ and apply it in a group of long standing diabetics in a cross-sectional manner to assess it applicability in clinical settings.

\section{Methods}

\section{Validation of the Sinhala version of $R A Q$}

We translated the original English version of RAQ to Sinhala using the standard backward and forward linguistic translation. After a face validation and piloting the Part A of the translated RAQ (SRAQPart A) was given to 47 adult patients who presented with chest pain. These patients had presented with chest pain of more than two weeks for which no obvious non-cardiac cause was apparent and the possibility of stable angina had been considered as the most likely possibility. The diagnosis of stable angina was confirmed when the resting ECGs showed evidence of myocardial ischaemia (dynamic symmetrical $\mathrm{T}$ inversions or $>2 \mathrm{~mm}$ ST depressions). Patients who had a normal resting ECG or changes non-confirmatory of myocardial ischaemia underwent treadmill examination to detect exerciseinduced myocardial ischaemia. Inducible myocardial ischaemia was confirmed when significant ST segment depressions $(>2 \mathrm{~mm})$ were found in one or more vascular territories. All Exercise-ECGs were done by the same technician adhering to one protocol and same cardiologist analysed them.

Part B of the translated RAQ (SRAQ-Part B) was given to a group of 68 patients who presented with acute chest pain of less than 24 hours where the possibility of acute coronary syndrome was suspected. These patients underwent serial ECGs and cardiac enzymes / troponin estimations. Fifty eight had acute coronary syndrome [ST elevated Myocardial Infarction (MI), non-ST elevated MI or unstable angina] confirmed by typical ECG changes and or elevated cardiac enzymes / troponin and the remaining 10 had alternative non-cardiac cause of chest pain detected by repeated clinical examinations or other investigations (radiography, ultrasonography or endoscopy) during the follow up period.

All the questionnaires were administered by a medical officer who had a prior knowledge on the rules applicable to filling the questionnaire. Angina and possible infarction were defined according to the WHO guidelines given with the original RAQ.

\section{Application of the Sinhala version of RAQ to a group of diabetics}

The validated Sinhala version of RAQ was administered to 311 diabetic patients attending medical clinics of Teaching Hospital, Karapitiya. These were the consecutive patients with adultonset diabetes of more than 6 months attending two medical units. Patients who were more than 80 years of age were excluded from the study.

None of the subjects participated in the validation of RAQ was included in this analysis.

\section{Results}

\section{Validation of the Sinhala version of $R A Q$}

The mean (SD) age of patients who filled the SRAQPart A was 54.4 (11.7) years and 21 of them were women. Eight had diabetes while 15 had hypertension. Patients who filled the SRAQ-Part B had mean (SD) age of 59.9 (13.9) years and 26 of them were women. The validation results of the two parts of the questionnaire are shown in the Table. Both parts of the Sinhala version of the RAQ were easily understood by patients. 
Table: Validation results of the Rose Angina Questionnaire

\begin{tabular}{|l|c|c|c|c|c|c|}
\hline Questionnaire & Sensitivity(\%) & Specificity(\%) & PPV(\%) & NPV(\%) & LR+ & LR- \\
\hline Part A & 86.4 & 76 & 76 & 86 & 3.6 & 0.18 \\
\hline Part B & 74.1 & 70 & 93.5 & 31.8 & 2.47 & 0.37 \\
\hline
\end{tabular}

PPV $=$ positive predictive value, $\mathrm{NPV}=$ negative predictive value, $\mathrm{LR}+=$ positive likelihood ratio, $\mathrm{LR}-=$ negative likelihood ratio

\section{Application of the Sinhala version of $R A Q$}

Mean (SD) age of diabetics included in this part was 62 (10.5) years and 97 were males. Mean (SD) duration of diabetes was 9.5 (5.8) years. Prevalence of hypertension, transient ischaemic attacks and cerebrovascular diseases were $66.7 \%, 19.7 \%$ and $3.3 \%$.

Of 311 patients with diabetes, 87 were test positive for the SRAQ Part A which is used to screen for stable angina. With $76 \%$ PPV, 66 of these (true positives) were likely to have stable angina. With $86 \%$ NPV, further 31 of 224 who answered negative (false negatives) for this part of the questionnaire were also likely to have the disease. Hence the prevalence of stable angina in this group of patients would be $31 \%(95 \% \mathrm{CI}=26-37 \%)$.

For the Part B of the questionnaire, 17 answered positively and rest negatively. Of these 17 positives, 16 were likely to have acute coronary disease (true positives). Since the negative predictive value of Part $\mathrm{B}$ of the questionnaire was very low we didn't calculate the number of false negatives. Among the 17 subjects who answered positive for part $\mathrm{B}, 10 \mathrm{had}$ answered positive for Part A as well. When the previous medical history was searched, of those who answered positive for part B, 10 were found to have suffered myocardial infarction whereas the remaining seven had no recorded previous acute coronary episodes. Only one among test negatives had acute coronary syndrome.

\section{Discussion}

Our analysis shows that the Sinhala translation of the full RAQ has high specificity and sensitivity in detecting either stable angina or acute coronary syndrome. Previous validations of RAQ have shown a wide variation in sensitivity and specificity values.
This probably occurred due to the variation in reference standards used. Studies which used clinician's diagnosis as the reference standard reported a sensitivity ranging from $25 \%-83 \%$ and a specificity of $48 \%$ - 98\% (9). Studies which used exercise treadmill test as the reference standard generally reported high specificity with variable sensitivity (6). We used clinically acceptable reference standards in confirming both stable angina and acute coronary syndrome and our analysis showed an acceptable sensitivity and specificity values.

A previous validation of RAQ in Sri Lanka using the exercise treadmill test as the standard to compare the results of translated modified RAQ, achieved a sensitivity of $80 \%$ and specificity of $31.2 \%$ (8). A Thai study which was conducted in a similar manner, however, achieved sensitivity of $30.3 \%$ and specificity $83.9 \%$ (6). In the present study we achieved comparatively higher sensitivity $(86.4 \%)$ and specificity (76\%) values to make it an acceptable tool for screening purposes among Sinhala conversant patients. The higher sensitivity we observed will help to "rule out" stable angina in a patient with a negative test while higher specificity will help to "ruling in" a positive patient.

Among 311 patients with diabetes, 31.2\% were detected to have stable angina according to the validated RAQ. This estimation is relatively high when compared to the values obtained in a previous study done in 1993 (10). The high prevalence of myocardial ischaemia could be explained by the characteristics of the sample. The present study included patients with a mean age of 62 years and also their mean duration of the disease was nearly 10 years. Another reason for the high prevalence could be the high prevalence of hypertension $(66.7 \%)$ which is an additional risk factor for myocardial ischaemia, among our cases. Furthermore, the study 
samples may vary with regards to the level of physical activity, social habits, lipid levels and other determinants of myocardial ischaemia.

This study has some limitations. The present study used exercise ECG as the gold standard. Coronary angiogram or Thallium Scintigram would have been more accurate gold standards. Using these methods was not practical in our setup due to financial constraints.

In conclusion this validated RAQ could be used as a screening tool for detection of stable angina or acute coronary episodes among Sinhala conversant local populations.

\section{Acknowledgement}

Prof. Sarath Lekamwasam, Professor in Medicine, Department of Medicine, Faculty of Medicine, University of Ruhuna for providing expert guidance and constant support throughout the study.

Staff members of Cardiology Unit, Teaching Hospital, Galle for their support.

University of Ruhuna Research Grant for providing financial support for the study.

\section{References}

1. Rose GA. The diagnosis of ischaemic heart pain and intermittent claudication in field surveys. Bull. Wld Health Org 1962; 27: 645-58.

2. Singh RB, Sharma JP, Rastogi V, et al. Prevalence of coronary artery disease and coronary risk factors in rural and urban populations of north India. Eur Heart J 1997; 18: 1728-35.
3. Kutty VR, Balakrishnan KG, Jayasree AK, Thomas J. Prevalence of coronary heart disease in the rural population of Thiruvananthapuram district, Kerala, India. Int J Cardiol 1993; 39: 59-70.

4. Reid DD, Brett GZ, Hamilton PJ, Jarrett RJ, Keen H, Rose G. Cardiorespiratory disease and diabetes among middleaged male Civil Servants. A study of screening and intervention. Lancet 1974; 1: 469-73.

5. Erikssen J, Forfang K, Storstein O. Angina pectoris in presumably healthy middle-aged men. Validation of two questionnaire methods in making the diagnosis of angina pectoris. Eur J Cardiol 1977; 6: 285-98.

6. Udol K, Mahanonda N. Comparison of the Thai version of the Rose questionnaire for angina pectoris with the exercise treadmill test. JMed Assoc Thai 2000; 83: 514-22.

7. Wilcosky T, Harris R, Weissfeld L. The prevalence and correlates of Rose Questionnaire angina among women and men in the Lipid Research Clinics Program Prevalence Study population. Am J Epidemiol 1987; 125: 400-9.

8. Nanayakkara SDI, Mendis S, Weerasinghe VS, Samarakoon C. Validation of the Sinhala version of the Rose questionnaire against the Exercise Electrocardiogram in a group of patients with chest pain. Sri Lanka Journal of Medicine 2002; 11: 8-17.

9. Garber CE, Carleton RA, Heller GV. Comparison of "Rose Questionnaire Angina" to exercise thallium scintigraphy: different findings in males and females. J Clin Epidemiol 1992; 45: 715-20.

10. Fernando DJ, Siribaddana S, Perera N, Perera S, de Silva D. The prevalence of macrovascular disease and lipid abnormalities amongst diabetic patients in Sri Lanka. Postgrad MedJ1993; 69: 557-61. 